\title{
KOJIMA ON DOUBLE DIRICHLET SERIES
}

\author{
BY C. R. ADAMS
}

The object of this note is to direct attention to the most extensive and for us one of the most interesting papers by Tetsuzô Kojima, whose death on April 7,1921, at the early age of thirty-four deprived Japan of an able and promising mathematician. This paper, which has only just now come to our notice* and whose existence may not be generally known, is On the double Dirichlet series, (Science Reports of the Tôhoku Imperial University, (1), (math., phys., chem.), vol. 9 (1920), pp. 351-400). It is devoted mainly to the convergence properties of double Dirichlet series, certain related double integrals, double factorial series, and double binomial coefficient series. Unaware of Kojima's work, Leja and the present writer $†$ have recently considered some of the same questions; it may therefore be appropriate to give a brief description of Kojima's memoir and to point out the main features of its relationship to the more recent papers cited.

A double series $\sum_{i, j=1}^{\infty} a_{i j}$ for which the sequence $\left\{S_{m n}\right\}$ of partial sums, $S_{m n}=\sum_{i, j=1}^{m, n} a_{i j}$, converges as $m, n \rightarrow \infty$ and of which each row and column converges has been called regularly convergent by Hardy. If $\left\{S_{m n}\right\}$ is both convergent and bounded, the series may be said to be convergent-bounded. One sees at once that every series regularly convergent is convergentbounded but that the converse is not true. The notion of regular convergence is basic in Kojima's work; that of convergencebounded in L, AI, and AII.

* An obituary in the Tôhoku Mathematical Journal, vol. 19 (1921), 2 pp. following the table of contents, gives a complete list of Kojima's fifteen papers, including two published posthumously, together with an account (in Japanese) of his life.

$\dagger$ Leja, Sur les séries de Dirichlet doubles, Comptes-Rendus du 1 Congrès des Mathématiciens des Pays Slaves, Warsaw, 1930, pp. 140-158;Adams, On multiple factorial series, Annals of Mathematics, (2), vol. 32 (1931), pp.67-82; and Adams, Note on multiple Dirichlet and multiple factorial series, ibid., vol. 33 (1932), pp. 406-412. Hereafter these papers will be referred to respectively as $\mathrm{L}, \mathrm{AI}$, and AII. 
Kojima begins (Theorem I and ensuing discussion) by es. tablishing the existence of related abscissas of regular conver. gence $S, T$ for the Dirichlet series

$$
\sum_{i, j=1}^{\infty} a_{i j} e^{-\lambda_{i} s-\mu i t},
$$

$\left\{\lambda_{i}\right\}$ being a sequence of real numbers monotonically increasing without limit and $\left\{\mu_{j}\right\}$ a like sequence. The existence of related abscissas of convergence-bounded is an immediate consequence of Theorem 1, part 1, of L, which is the same as Theorem 1 of AII. That a pair of abscissas of regular convergence is also a pair of abscissas of convergence-bounded, and conversely, follows at once from remarks in both L and AII. The precise significance of having one abscissa finite and its related abscissa infinite is specified only by Kojima.

In Theorem II, Kojima gives for double series a generalization of his own formula for the convergence abscissa, not necessarily positive, of a simple Dirichlet series.* Theorem III, stating a necessary and sufficient condition that a pair of numbers $S, T$, neither necessarily positive, be related abscissas of regular convergence, follows easily. A similar investigation of pairs of related positive abscissas, suggested by the Cahen formula for the convergence abscissa (positive) of a simple Dirichlet series, is made in L, the results being contained in Theorems III, IV, and V. Regarding $T$ as a function of $S$, Kojima then proves the convexity of this function and subjects it to a careful further analysis. This is paralleled, in part, by Theorem 6 of $\mathrm{L}$ and the attendant discussion.

Theorem IV is an analog of Theorem II for absolute convergence. Theorem $\mathrm{V}$, obviously mis-stated, should be given the following form: If $S, T$, is any pair of related abscissas of regular convergence, there always exists a pair of related abscissas of absolute convergence $\bar{S}, \bar{T}$ satisfying the inequalities

$$
\vec{S}-S \leqq \limsup _{m \rightarrow \infty} \frac{\log m}{\lambda_{m}}, \quad \vec{T}-T \leqq \limsup _{n \rightarrow \infty} \frac{\log n}{\mu_{n}} .
$$

Theorem VI contains a generalization of Fujiwara's formula for

* This formula, given in 1914 , he had shown to be a natural extension of the Cauchy-Hadamard formula for the radius of convergence of a power series. 
the convergence abscissa (not necessarily positive) of a simple Dirichlet series.

Extensions of theorems of Vitali on sequences of analytic functions are given as Theorems VII and VIII. Theorem IX, concerning uniform convergence in a finite region and the consequent analyticity of the function represented, is essentially duplicated by Theorem 1, part 2, of $\mathrm{L}$, but is less general than Theorem 2 of AII on uniform convergence for an infinite region. Theorem $\mathrm{X}$ asserts that if the coefficients in (1) are all positive, $(S, T)$ is a singular place for the function represented. Theorem $\mathrm{XI}$, on simple Dirichlet series, is a preliminary to the proof of Theorem XII on the convergence of (1) in the region $R(s)=S$, $R(t)=T$.

Theorem XIII, on regular convergence of a double factorial series, is slightly less general than Theorem 1 of AI as supplemented by remarks on page 70 of $\mathrm{AI}$ and strengthened on page 407 of AII. Theorem XIV, on the identity of the related halfplanes of regular convergence for a factorial series and its associated special Dirichlet series, is duplicated by Theorem 3 of AII. Theorem $\mathrm{XV}$, on uniform convergence of a factorial series in a finite region, is slightly less general than the similar Theorem 2 of AI and much less general than Theorem 7 of AI (supplemented by footnote 12 of AII) on uniform convergence for an infinite region. Theorems XVI, XVII, and XVIII are respective analogs for binomial coefficient series of the preceding three theorems on factorial series.

BROWN UNIVERSITY

\section{A CENSUS OF SQUARES OF ORDER 4, MAGIC IN ROWS, COLUMNS, AND DIAGONALS*}

BY D. N. LEHMER

This census was made by Frenicle in 1693 who found 880 fundamental squares which with their rotations and reflections gave 7,040 squares of this sort. A study of the transformations which throw a square of this type into another of the same type shows that Frenicle need not have listed more than 220 fundamental squares. Besides his transformations, which were a rotation $R$ of order 4 of the square in its plane about a right angle,

* Presented to the Society, December 2, 1933. 